\title{
Kaleidoscope Lasers - Complexity in Simple Optical Systems
}

\author{
J. M. Christian and G. S. McDonald \\ Joule Physics Laboratory, School of Computing, Science and Engineering, \\ Materials \& Physics Research Centre, University of Salford, Salford M5 4WT, U.K. \\ J. G. Huang \\ School of Engineering and Mathematical Sciences, City University London, London EC1V OHB, U.K.
}

\begin{abstract}
We present the first detailed account of modelling kaleidoscope laser modes where the equivalent Fresnel number $N_{\text {eq }}$ and magnification $M$ may assume arbitrary values. The convergence toward circularity is also investigated through extensive numerical computations.

(C) 2010 Optical Society of America

OCIS codes: (140.3410) Laser resonators; (050.1970) Diffractive optics.
\end{abstract}

\section{Introduction}

Unstable cavity lasers exhibit a plethora of bizarre and potentially exploitable phenomena that have fascinated researchers over the last four decades. In particular, the innate capacity of such simple systems for generating complex light patterns continues to attract wide and sustained interest. In the late 1990s, Karman and Woerdman [1] found that the eigenmodes of one-dimensional (1D) confocal resonators are fractals - patterns that exhibit comparable levels of detail spanning many scalelengths. The fractality of these self-reproducing mode profiles was later shown to originate in the interplay between periodic aperturing and diffraction at the outer boundary of the system (i.e., the feedback mirror) [2].

Kaleidoscope lasers are intuitive generalizations of the classic unstable strip resonator to $2 \mathrm{D}$ transverse geometries where the feedback mirror has the shape of a regular polygon [3]. The non-orthogonal edges of the aperturing element have a profound impact on the structure of the cavity eigenmodes, which exhibit a striking level of beauty and complexity (see Fig. 1). Most obviously, $N$-sided regular-polygon boundary conditions impose $N$ fold rotation symmetry on the intensity pattern. Kaleidoscope lasers offer enormous prospects for inspiring novel photonic device designs and architectures. For example, fractal light may prove to be more efficient for probing, scanning and ablation applications in various technological, medical and industrial contexts.

Here, we present the first detailed analysis of kaleidoscope lasers with arbitrary equivalent Fresnel number $N_{\text {eq }}$ and magnification $M$. All previous analyses have been restricted to regimes where either $N_{\text {eq }}=O(1)$ (in which case conventional ABCD matrix modelling can be deployed [4]) or $N_{\mathrm{eq}} \gg O(1)$ (where asymptotic approximations can be used [5]). Our approach is based on a fully-2D generalization of Southwell's Virtual Source method [6], and exploits exact mathematical descriptions of the constituent Fresnel diffraction patterns [7]. One key advantage of our technique it that a single calculation allows one to access entire families of modes; another is that any particular mode may be computed to any desired accuracy. We also quantify the convergence properties of kaleidoscope lasers - the eigenvalue spectra and the mode patterns themselves - in the limit $N \rightarrow \infty$ (where the feedback mirror becomes circular).
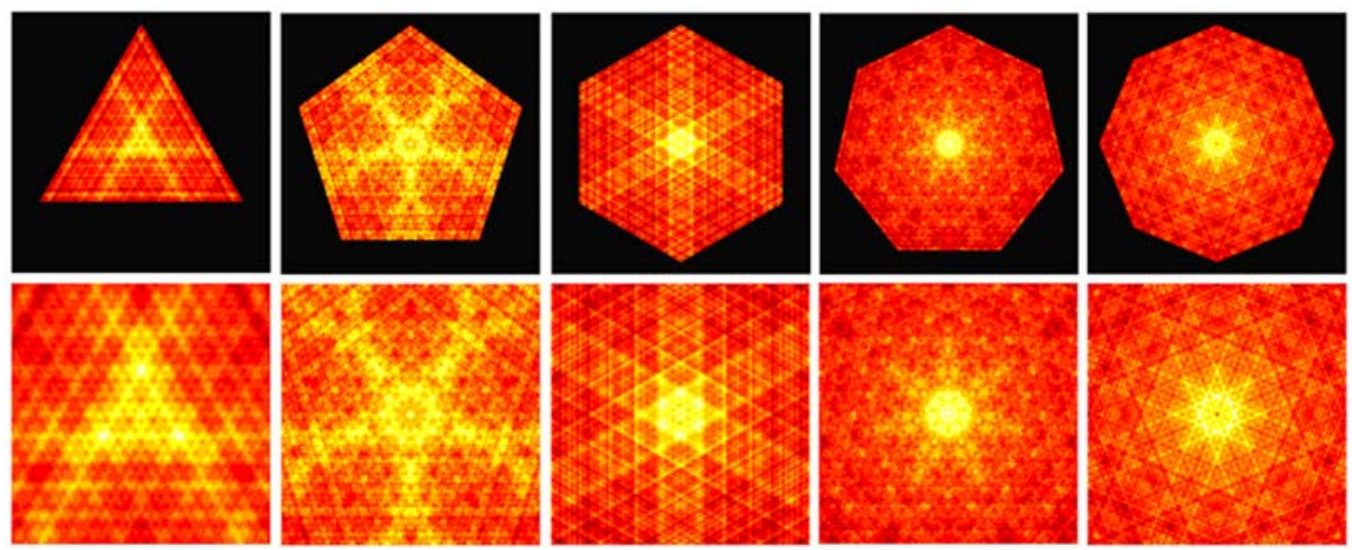

Fig. 1. Lowest-loss eigenmode patterns for kaleidoscope lasers with $N_{\mathrm{eq}}=30$ and $M=1.5$. 


\section{Modelling kaleidoscope lasers}

The virtual source method [6] unfolds an unstable cavity into its equivalent sequence of $N_{S}=\log \left(250 N_{\mathrm{eq}}\right) / \log M$ virtual apertures. Any eigenmode can then be constructed from a weighted superposition of the edge-waves diffracted by each aperture, plus a plane-wave contribution. In scaled units, the mode pattern $V(\mathbf{X})$ is given by

$$
V(\mathbf{X})=e_{0}\left[\frac{E_{N_{S}+1}\left(\mathbf{X}_{\mathbf{C}}\right)}{\alpha^{N_{S}}(\alpha-1)}+\sum_{m=1}^{N_{S}} \alpha^{-m} E_{m}(\mathbf{X})\right]
$$

where $\mathbf{X}$ denotes an appropriate set of transverse coordinates, $\mathbf{X}_{\mathbf{C}}$ is any point on the boundary of the feedback mirror, and $E_{m}(\mathbf{X})$ is the edge-wave pattern arising from the $m^{\text {th }}$ virtual aperture [7]. Since our interest lies with calculating the field over the feedback mirror, $e_{0}=+1$ if $\mathbf{X}$ lies inside this domain, and $e_{0}=0$ otherwise. The weighting factor $\alpha$, which plays the role of the mode eigenvalue, is obtained by finding the roots of an associated polynomial equation. A single virtual-source calculation gives one immediate access to entire families of modes.

\section{The circular limit of a kaleidoscope laser}

When $N \rightarrow \infty$, the feedback mirror becomes circular and the cavity essentially has only a single transverse dimension. This limit has been investigated by Berry under the assumption $N_{\text {eq }} \gg O(1)$, and only for the lowestloss mode [8]. For cavities with arbitrary $N_{\mathrm{eq}}$ and $M$, this type of fully-2D convergence phenomenon does not lend itself to asymptotic analysis; indeed, it can only be truly addressed via numerical computation (see Fig. 2). We will present, to the best of our knowledge, the first in-depth treatment of the circular limit of families of kaleidoscopelaser modes. This is a far more subtle problem than might first be imagined.
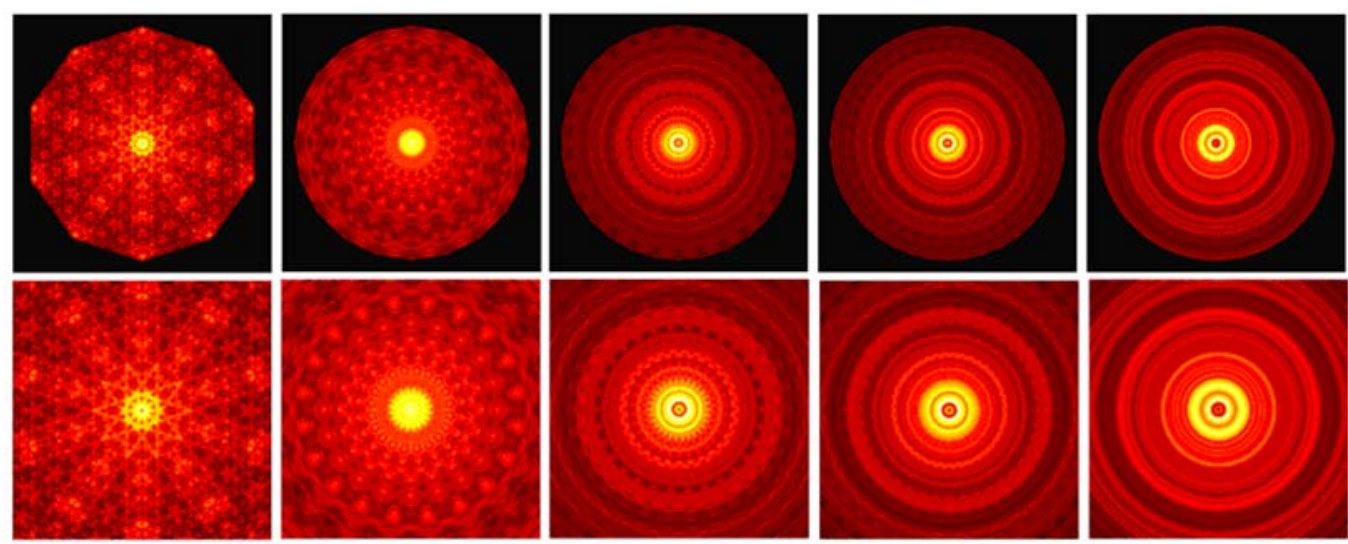

Fig. 2. Convergence of the lowest-loss kaleidoscope laser with $N_{\mathrm{eq}}=30$ and $M=1.5$ toward its circular counterpart. Going from left to right: $N=10,20,30$ and 40 . The fifth column is the circular target pattern.

\section{References}

[1] G. P. Karman and J. P. Woerdman, “ Fractal structure of eigenmodes of unstable-cavity lasers,” Opt. Lett. 23, 1909-1911 (1998).

[2] G. H. C. New et al. "Diffractive origin of fractal resonator modes," Opt. Commun. 193, 261-266 (2001).

[3] G. P. Karman, G. S. McDonald, G. H. C. New and J. P. Woerdman, "Fractal modes in unstable resonators," Nature 402, 138 (1999).

[4] G. S. McDonald, G. P. Karman, G. H. C. New and J. P. Woerdman, “Kaleidoscope laser,” JOSA B 17, 524-529 (2000).

[5] M. Berry, C. Storm and W. van Saarloos, “Theory of unstable laser modes: edge waves and fractality,” Opt. Commun. 197, 393-402 (2001).

[6] W. H. Southwell, "Virtual-source theory of unstable resonator modes," Opt. Lett. 6, 487-489 (1981).

[7] J. G. Huang, J. M. Christian and G. S. McDonald, "Fresnel diffraction and fractal patterns from polygonal apertures," JOSA A 23, $2768-2774$ (2006).

[8] M. Berry, "Fractal modes of unstable lasers with polygonal and circular mirrors," Opt. Commun. 200, 321-330 (2001). 